\title{
"Sometimes You Reach a Point Where You Feel Burnt Out", Job Satisfaction and Psychosocial Well Being of COVID-19 Frontline Health Workers: a Qualitative Study in Two Ugandan COVID-19 Treatment Centres.
}

Twinamasiko Nelson ( $\square$ drtwinamasikonelson@gmail.com )

Makerere University https://orcid.org/0000-0003-3716-1468

Adelline Twimukye

Makerere University Infectious Diseases Institute

Anna Maria Gwokyalya

Makerere University Faculty of Medicine: Makerere University College of Health Sciences Innocent Nakityo

Makerere University Faculty of Medicine: Makerere University College of Health Sciences

\section{Enock Wasswa}

Makerere University Faculty of Medicine: Makerere University College of Health Sciences

Emmanuel Sserunjogi

Makerere University Faculty of Medicine: Makerere University College of Health Sciences

Mohammed Lamorde

IDI: Makerere University Infectious Diseases Institute

Harriet Kizza Mayanja

Makerere University Faculty of Medicine: Makerere University College of Health Sciences

Pius Rwamafa

Mulago Hospital: Mulago National Referral Hospital

\section{Research}

Keywords: COVID-19, Psychological well-being, Frontline Healthcare workers, Job satisfaction, qualitative research, Uganda

Posted Date: January 25th, 2021

DOI: https://doi.org/10.21203/rs.3.rs-153208/v1 
License: (c) (i) This work is licensed under a Creative Commons Attribution 4.0 International License. Read Full License 


\section{Abstract}

BACKGROUND: COVID-19 has become a major global health challenge, with Uganda reporting over 25,000 cases. Frontline healthcare workers (HCWs) are the most at risk population for mental health disorders yet their well-being is key to combating the pandemic. We explored the psychosocial wellbeing and job satisfaction of COVID-19 Frontline HCWs in Uganda.

METHODS: This was a qualitative study done at Entebbe regional Referral hospital (ERRH) and Mulago National Referral hospital (MNRH) in September 2020. Data collection was through 3 Focus Group Discussions (FGDs) with 5 participants for each FGD. 2 FGDs were at MNRH and 1 at ERRH. Participants included; doctors, nurses, laboratory personnels, hygienists and a security personnel. Interviews were audio-recorded, transcribed and analyzed thematically using Nvivo version 12 software.

RESULTS: Despite the challenges HCWs faced, they were motivated to work when they saw patients recover and go home safely. Participants felt the Pandemic was unprecedented and as a result no person or government was fully prepared. Big unexpected patient crowds caused limited and/ or inconsistent medical supplies. Additionally, Poor Procurement lines affected sufficiency of medical supplies and equipment such as medical gowns, drugs, PPE, alcohol, beds, and COVID-19 testing kits. There was knowledge gap among the HCWs regarding COVID-19 management. This put the staff in a Panic situation hence practicing "trial and error" treatment. Poor remuneration in terms of low or delayed Salary, lack or delay of risk allowance caused dissatisfaction among staff and were affected mentally given that they were involved in risky work yet their families were suffering economically.

CONCLUSION: HCWs were remarkably stressed, exhausted and burnt-out due to the heavy workload and inadequate personal protective Equipment. These findings depict a need of creating a conducive environment for these HCWs. Government and ministry should re-strategize on how well to take care of Covid 19 frontline HCWs to save lives.

\section{Introduction}

Coronavirus disease 2019 (COVID-19) is outlined as an illness caused by a coronavirus, currently known as Severe Acute Respiratory Syndrome coronavirus 2. COVID-19 was first identified in Wuhan city, China in late December, 2019 and has since been identified as a disease that is rapidly transmitted between people $(1,2)$. The most common manifestations of COVID-19 in a person have been discovered as being difficulty in breathing, fever, tiredness, muscle aches and pain, and dry cough (3). In order to mitigate its quick spread, World Health Organisation had declared COVID-19 a public health emergency of international concern by the start of February 2020 and thus calling for a conjoint effort from all countries (4). COVID-19 has led to remarkable economic, social, and psychological havoc for individuals and nations as well.(5)

Health workers are the pandemic's frontliners and them working effectively and normally is vital to the effective pandemic response.(6) In these situations, the health workers are crucial reference points for 
citizens as to how best the pandemic can be contained.(7) This in a way might bring a perception among most of the health workers that they are fully in charge of controlling the situation which in many times hinders their ability to acknowledge their own concerns and feelings thus creating a lot of stress and emotional drain. $(8,9)$

Earlier encounters and managing of pandemics have shown that for healthcare workers to be efficient, they should be supported and encouraged to be mindful of their own emotions by sharing their experiences.(10)

As of 4th December 2020, the Government of Uganda had reported a cumulative total of 21,406 COVID19 cases among nationals.(11) Among Ugandans, there had been 27 cases in health care workers.(11) Italy being one of the most affected countries, as of 16th April 2020, 16,991 healthcare workers had been reported by the Italian National Institute of Health to be infected with COVID-19 with an estimated death of 119 medical doctors.(12) With the increase in the number of COVID-19 cases, the numbers of healthcare workers infected increase as well.

A study done by Xong and Peng in china revealed that the increase in covid-19 cases and mortality due to the pandemic brought extreme pressure to the frontline healthcare workers due to many factors that included: stigma, social isolation and discrimination; which subsequently placed them at a threat of psychological problems.(13) Furthermore, a study done by Lai and colleagues revealed that a considerable number of healthcare workers who managed COVID-19 patients had anxiety, distress, and depression.(14)

Generally, COVID-19 Frontline healthcare workers are the most at risk population for mental health disorders yet their well-being is key to combating the pandemic. Both immediate and prolonged consequences of global infectious disease outbreaks on the mental health of health care workers is irrefutable, however, currently in Uganda, the mental well-being of frontline healthcare workers has been of little focus.

\section{Methods}

\section{Study Design}

The study was a cross-sectional survey using qualitative method of data collection.

\section{Study site and setting}

The study was carried out in two COVID-19 treatment facilities selected basing on proximity and convenience of the study staff. The treatment facilities were; Mulago National Referral Hospital (MNRH) and Entebbe regional referral hospital (ERRH). MNRH and ERRH, both are the main referral hospitals for complicated COVID-19 cases and they have managed atleast half of Uganda's COVID-19 cases. 
ERRH is located in the central business district of the town of Entebbe, in Wakiso District, approximately 44 kilometers, by road, southwest of MNRH, the largest hospital in the country, located in Kampala, Uganda's capital and largest city. Each of the two hospitals has over 150 healthcare workers involved directly and indirectly in managing COVID-19.

Frontline healthcare workers (laboratory personnels, nurses, midwives, medical doctors, and supporting staff like hygienists, security personnels and nutritionists) in the two institutions directly involved in managing COVID-19 cases were recruited in this study.

\section{Sampling}

All the 15 participants that got involved into the study were recruited on a purposeful and convenience based sampling based on inclusion and exclusion criteria.

\section{Inclusion and Exclusion criteria}

- Health workers that were 18 years and above, and gave consent to participate in the study were allowed to participate.

- Frontline health workers who did not give consent, and those severely ill were excluded from participating in the study

\section{Data Collection}

Focal persons who were part of the study staff mobilized the healthcare workers (HCWs). All the HCWs that were approached by the focal persons were made aware of the study and that participation was entirely voluntary and confidential.

Focus group discussions (FGDs) were employed for this study. FGD is an accredited tool for collection of qualitative data appropriate for research studies aiming at exploring the thoughts and feelings of people where importance is on investigating people's experiences and opinions.(15) Three FGDs were done, one from ERRH and two from MNRH with each FDG containing 5 participants. Among the participants included: a doctor, nurse, laboratory personnel, Pharmacist, or security personnel. The participants were chosen randomly basing on their convenience.

The discussion was moderated by one of the study staff using a topic guide including open ended questions and the moderator started by asking about their motivation for joining COVID-19 frontline work, followed by questions on experience and challenges while managing COVID-19 patients, and lastly questions on the possible solutions for the mentioned challenges. Additional file 1 contains the FGD guide. Interviews were conducted in English by NT, AG, and IN and the sample size was determined by saturation. FGDs were audio-recorded with participants consent and transcribed verbatim for data analysis. AT checked the transcripts for completeness and accuracy.

\section{Data Analysis.}


Verbatim transcription of the audio-recorded data was done. Data was organized and managed using Nvivo software Version 12. The initial codebook was developed after carefully analyzing the text line-byline of each transcript through open coding. We assigned codes to relevant segments of the text, and similar or related codes aggregated to form themes. Themes/codes were derived in relation to the research questions/objectives and literature. A narrative was generated from the dominant themes. Some quotes are used to represent in the narrative.

\section{ETHICAL CONSIDERATION.}

The study sought permission from Mulago Hospital Research and Ethics Committee under protocol number MHREC 1906. Permission was further got from administration of all the two respective facilities.

A consent form was given to every participant before being involved into the study. Whoever gave consent after being fully informed about the study, was thereafter allowed to participate. The study was entirely voluntary and whoever wanted to withdraw, did so with no repercussion. Confidentiality was highly observed as participants were only identified by numbers rather than their names.

\section{Results}

A total of 15 participants were involved in this study. Of these majority were males (53.3\%) and the majority age range was 25-34 years. Regarding Job security, majority of the participants were on short term contracts $(66.7 \%, n=10)$. Table 1 presents the socio-demographic characteristics of the participants. 
Table 1

Characteristics of participants.

\begin{tabular}{|lll|}
\hline Demographics (N= 15). & Frequency & $\%$ \\
\hline Age in years & & \\
\hline $18-24$ & 03 & 20.0 \\
\hline $25-34$ & 07 & 46.7 \\
\hline $35-44$ & 03 & 20.0 \\
\hline$\geq 45$ & 02 & 13.3 \\
\hline Sex & & \\
\hline Female & 7 & 46.7 \\
\hline Male & 8 & 53.3 \\
\hline Marital status & & \\
\hline Married & 9 & 60.0 \\
\hline Single & 5 & 33.3 \\
\hline Divorced & 1 & 6.7 \\
\hline Widowed & 0 & 0.0 \\
\hline Occupation & & 20.0 \\
\hline Hygienist & 03 & 13.3 \\
\hline Nurse & 03 & 20.0 \\
\hline Pharmacist & 03 & 20.0 \\
\hline Security Personnel & 01 & 6.7 \\
\hline Doctor & 03.7 \\
\hline Laboratory Personnel & 03 & \\
\hline Job Security & & \\
\hline Permanent contract & 02 & \\
\hline Short term contract & 10.0 \\
\hline Volunteer & & \\
\hline
\end{tabular}

From the focus group discussions held, four major themes were obtained. These were (1) Frontline health workers' attitudes towards covid 19, (2) Psychological challenges among covid-19 frontline health 
workers, (3) Barriers to job satisfaction among covid 19 frontline health workers, and (4) Suggestions to support people working during covid-19 pandemic.

\subsection{Frontline health workers' attitudes towards covid 19.}

Frontline health workers in all the three FGDs comprised of contract and permanent staff. They included; doctors, nurses, laboratory personnels, hygienists and a security personnel. Several frontline health workers said they felt an obligation to work on COVID-19 patients to fulfil the Hippocratic Oath in which they swore to save patients despite the stress they undergo:

When we finish our studies, we swear the Hippocratic Oath that we must serve. We are supposed to attend to these patients because it is our work... But also the healthcare professional must also be willing to be able to help others because that is the whole point of being a professional. (FGD_Covid Frontline Worker_COVID-19 Treatment Unit Mulago Hospital _Participant (P4))

Most frontline workers said they treated patients with compassion. They were happy to see patients recover and go home safely which steered their morale to keep helping in a novel disease.

These last two weeks, we have been having quite a number of very ill patients. Managing them up to the day of discharge is a high for me. Delivering the COVID-19 pregnant mother, the very first COVID-19 mother in the country, it was a high for me as well. (FGD_ Covid Frontline Worker Entebbe Regional Referral Hospital_P2)

There's nothing as interesting as seeing someone who came dying moving out while smiling and calling your name. This is someone who might have tasted death but he sees you've been with him there and then. (FGD_ Covid Frontline Worker Entebbe Regional Referral Hospital_P1)

The value of staff maintaining psychosocial wellbeing, physical health, having good attitude, and good relationship with patients was deemed as critical during work. Patients appreciated the care frontline workers offered and that motivated HCWs because they felt their work was valued:

There have been some happy times, times when patients recognize and call you back and are very thankful. Being nice to us, makes us remember that what we're doing is worth it and being appreciated. (FGD_Covid Frontline Worker Entebbe Regional Referral Hospital_P2)

\subsection{Barriers to job satisfaction among covid 19 frontline health workers.}

\subsection{Health system barriers}

Most frontline workers felt the Pandemic was unprecedented and as a result no person or government was prepared to fully manage COVID-19:

No one prepares for a pandemic but I want to give ministry and government credit because they have done their duty though they are not yet there. They have done their best but I will not say they are yet 
there. (FGD_Covid Frontline Worker_COVID-19 Treatment Unit Mulago Hospital_P2)

Overwhelming unexpected patient crowds caused limited and or inconsistent medical supplies and equipment. Poor medical infrastructure such as limited beds and lack of ventilators at ICU was reported by some participants:

So because of the increasing numbers and the challenges that the pandemic has brought to the procurement plans, definitely there are some shortages. (FGD_Covid Frontline Worker_COVID-19 Treatment Unit Mulago Hospital_P1).

Previously we have been having patients in ERRH sleeping on the ground. If they were sleeping on the ground, were they even keeping the $2 m$ distance? (FGD_Covid Frontline Worker_COVID-19 Treatment Unit Mulago Hospital_P3).

I think COVID-19 exposed the gap in Uganda's health system. Before COVID-19, there were like probably close to 200 active ICU beds in the country, that's including all the private ones. (FGD_Covid Frontline Worker Entebbe Regional Referral Hospital_P5).

Most frontline health workers including doctors said it was not practically possible for them to enter COVID-19 treatment rooms or touch a patient without PPE. This was because they feared being infected. They said, government provided N95 masks and sanitizers but they were few:

For example when I come and there is no PPE, I cannot dare enter those treatment rooms. For me to work, PPEs must be there. I take it that my life comes first and other things come later. (FGD_Covid Frontline Worker_COVID-19 Treatment Unit Mulago Hospital_P1).

Poor Procurement lines affected sufficiency of medical supplies and equipment such as medical gowns, facemasks, and non-re-breather masks for patients who were desaturating, drugs, Personal protective equipment (PPE), alcohol, beds, ambulances as well as COVID-19 testing kits:

Procurement lines have been tampered with. They have been messed up. So because of that getting medical gowns, drugs etc. is a challenge. (FGD_Covid Frontline Worker_COVID-19 Treatment Unit Mulago Hospital_P1)

Participants said they identified "COVID patient champions" to talk to fellow patients when they lacked PPE to attend to patients. This made them feel that they were not doing a good enough job because the patients needed care from them not fellow patients.

We have been identifying patients' champions among patients themselves who have been doing some work like talking or calling patients which some patients have been taking it in good spirit and some others thinking that we are giving them our roles to play. They don't understand what we have been through and we cannot just come and say, 'We cannot attend to you because we do not have ABC,' because they will be calling people outside that we are lamenting. So we keep quiet while trying to find 
ways of making sure that work is done. (FGD_Covid Frontline Workers_COVID-19 Treatment Unit Mulago Hospital_P1)

Frontline workers were challenged with stock outs of drugs. Most of the drugs were not on essential drug list yet outsourcing was difficult:

I work in the pharmacy department, so there are some drugs the national referral doesn't have on their essential drug list, however they are prescribed by the doctors so because of that as the pharmacy department, we are tasked to get medication from outside. Sourcing from out was a little bit difficult, still is a little bit difficult but we are handling it. (FGD_ Covid Frontline Workers_COVID-19 Treatment Unit Mulago Hospital_P3)

\subsection{Facility related barriers.}

Moreover, due to limited research in COVID-19, all healthcare workers including doctors, nurses and the management team doubted the treatment given to patients. They lacked the confidence to treat COVID-19 patients. There was knowledge gap among the health workers regarding COVID-19 management guidelines and plans. This put the staff in a Panic situation where they practiced "trial and error" treatment given they were uncertain about the effectiveness of the treatment. Participants Suggested evidence based information to determine treatment basis:

The management plans we are using are not evidence based. We are just trying out things. We are in a panic, trying out things here and there. Some treatment works in some people and in others it doesn't work. Yeah so really, it is still challenging. We are not doing our best because we don't have evidence. (FGD_Covid Frontline Workers_COVID-19 Treatment Unit Mulago Hospital_P2).

The participants felt treating COVID-19 was challenging because it is a new illness that affected many people in the society yet the staff were limited:

There is a lot of understaffing here. I feel like we do not have an intensivist. Imagine we had ICU patients, who would look after them? We don't have that yet. We only have one physician on fulltime, so if he needs a break, what will happen? (FGD_Covid Frontline Worker Entebbe Regional Referral Hospital_P5).

Majority of Participants reported about long turnaround time of COVID-19 test results due to the overwhelming numbers and Stock out of testing kits. Long turnaround time which is 2-3 days results in increased risk of transmission of COVID-19 among the population according to participants in all FGDS. Long turnaround time for test results causes patients to overstay in hospital which causes their anxiety and stress. One participant said, Plans are in place to get gene Xpert testing to receive test results within 2 hours. Frontline workers Suggested reduction in turnaround time for results (30 minutes);

The turnaround time for covid results is 24 hours outside Mulago but in Mulago its $48-72$ hours. The reason for the delay is partly because of overwhelming numbers but also because of the modality (DNA- 
PCR). If it were rapid diagnostic tests like those for Malaria and HIV on the market, it would have been shorter probably. (FGD_Covid Frontline Workers_COVID-19 Treatment Unit Mulago Hospital_P5).

Poor remuneration in terms of low or delayed Salary, lack of or delay of risk allowance caused dissatisfaction among staff. This affected staff mentally given they were involved in risky work yet their families were suffering economically:

Limited and delayed salary definitely causes dissatisfaction and demoralizes the general staff because we come here every day to risk our lives yet we are not remunerated. We have personal needs and families to take care of. (FGD_C Covid Frontline Workers_COVID-19 Treatment Unit Mulago Hospital_P3).

Delays of salaries were reported by most participants. They felt demoralized because it was hard for them to go to work:

So far I have spent more than one and half months without being paid, so if it was because of money, I would not be here. But because I swore the Hippocratic Oath, patients' lives come first. (FGD_Covid Frontline Workers_COVID-19 Treatment Unit Mulago Hospital_P3).

Most Participants said they suffered transport constraints while coming for duty due to delay and limited money in form of allowances:

Of course we are not satisfied. Some of us are not getting even the transport and other allowances. But of course that is the reality we are dealing with right now yet we are the ones that risk more. You come work and you are not facilitated as you should be. (FGD_Covid Frontline Workers_COVID-19 Treatment Unit Mulago Hospital_P2).

Corruption was reported by one participant who suggested that if government releases money, the anticorruption unit should intervene to follow up that money such that it does what its intended for. There was a feeling among participants that their allowances and salaries were shadowed somewhere in politician offices:

People are demotivated but they have to be there because those big bosses intimidate them. They need to be here but you are not providing the necessities for people to be at work. (FGD_COVID Frontline Workers_COVID-19 Treatment Unit Mulago Hospital_P1).

\subsection{Psychological challenges among covid-19 frontline health workers.}

\subsection{Fear and anxiety of contracting Covid while on duty.}

Most participants reported fear and anxiety of contracting COVID-19 while on duty because several people were dying in countries like Italy, however this has reduced over time:

At first, you could find that when I sneeze I'm like eh, have got COVID. But I overcame that. At first, I was at tension, but now I'm free surely. (FGD_ Covid Frontline Workers_COVID-19 Treatment Unit Mulago 
Hospital_P1).

\subsection{Fear to spread Covid to family members.}

Family members were scared of acquiring COVID-19 from the frontline workers. Some tried to talk staff out of the COVID-19 work. It was a stressful moment for frontline workers who isolated and avoided their family members:

My family members initially were scared and tried to talk me out of it but later they became okay with it. So whenever I'm here, I try as much as I can to use the protective gears. (FGD_ Covid Frontline Workers_COVID-19 Treatment Unit Mulago Hospital_P1).

Most participants were scared about infecting family members and neighbours with COVID-19. As a result, they had to get separate places where they could stay alone to avoid mixing with family members and friends commonly expressed as; "I won't even meet you, I fear getting COVID":

When my family got to know that I'm going to be in COVID-19 treatment centre, they got scared. They wanted to tell me that I stay here at the hospital and not come back home because they were scared that I might infect them. I tried to tell them that we take all the necessary precautions to prevent infection but of course I could not convince them. Because of that, me I do not go home. I got somewhere to stay alone without mixing with my family members. (FGD_Covid Frontline Workers_COVID-19 Treatment Unit Mulago Hospital_P2)

I told my wife to arrange supper and go to the bedroom while I was sleeping in the sitting/dining room. I told her to make sure that she puts water and soap outside for me to bathe and wash my clothes I'm putting on because of that fear. (FGD_C Covid Frontline Worker Entebbe Regional Referral Hospital_P3).

\subsection{Stress as a result of avoidance or isolation from friends and family members.}

Most Participants said they missed their family members and friends due to avoidance or isolation as a result of working in a COVID-19 setting:

Psychologically, this is a disease that requires you to be in one place, so there's missing of your family, your loved ones and as you know ladies demand a lot of time, children need time, so that one also was a stress factor. (FGD_Covid Frontline Worker Entebbe Regional Referral Hospital_P3).

\subsection{Burn out as a result of heavy work load.}

Most Participants mainly from Entebbe reported stress, exhaustion, and burnout due to heavy workload, working long hours and days without day offs:

Also the burnout, you may burn out yet there's no one to leave on station, so it psychologically tortures you. You find you have an off but you can't even get to enjoy it because you'd be punishing a client. (FGD_Covid Frontline Worker Entebbe Regional Referral Hospital_P2). 
It's been stressful for some time. I think long working hours and longer days have impacted. Sometimes you reach a point where you feel a burn out, you're dragging your feet to work. You know there are times I really thought I had COVID-19, 3 to 4 times. (FGD_Covid Frontline Worker Entebbe Regional Referral Hospital_P2).

\subsection{Stigma and discrimination.}

Most frontline health workers suffered Stigma and discrimination associated with working on COVID-19 patients. There was a lot of stigma attached to COVID19 and as a result community members and neighbours kept a distance when they got to know one was a frontline worker.

Stigma was very apparent in the first days when the disease was quite novel and the news that was breaking seemed to be very precarious. So at the time people would stigmatize it. When getting transportation to work, you would say you are going to Mulago, they would say do you have a card, you show your identity card only for them to leave you on the side of the road. (FGD_Covid Frontline Worker Entebbe Regional Referral Hospital_P3).

Children of one participant were isolated from others because their parent was working as part of the COVID-19 team:

It shocked me when I went home and people started ignoring me. Actually they even started ignoring my kid at home, not allowing her to play with theirs, so they were isolated. So I'd tell the mum to lock her in the house and she'd cry. So it was still a bit of a challenge. (FGD_ Covid Frontline Worker Entebbe Regional Referral Hospital_P5)

Fear of stigma was the reason why most participants did not disclose to their neighbours about their workplace because they feared pointing fingers at them:

For me, the apartment where I stayed, I must say I took it deliberately not tell them (neighbours) because people were very scared about the disease and there was a lot of stigma. So If I had told them that I work in the COVID unit, I don't know what would have been their reaction but I think it would not have been good. (FGD_Covid Frontline Workers_COVID-19 Treatment Unit Mulago Hospital_P3).

\subsection{Stress associated with handling critical patients.}

Handling critical patients affected some participants mentally. They expressed need for stress management to be able to carry out their normal work efficiently:

I think it is part of continual growth. Mentally of course, there are times when you feel you need some time to digest everything around but of course, most of us have gone through worse days, treating critical patients. However, there are times when you feel you have broken down and you need some time to reassess and energize yourself. (FGD_ Covid Frontline Workers_COVID-19 Treatment Unit Mulago Hospital_P5) 
Actually, the first case in Uganda tortured me as a person because when the results came back and showed it was the first positive in Uganda, I was shocked as a human being. That very day I went home because the administration had not yet got for us accommodation. (FGD_ Covid Frontline Workers_COVID-19 Treatment Unit Mulago Hospital_P5).

\subsection{SUGESTIONS TO SUPPORT PEOPLE WORKING DURING COVID-19 PANDEMIC}

\subsection{Government Level}

Government relocate budget resources to COVID-19 medical supplies and avail all infrastructure needed in the CTU (COVID-19 Treatment Unit). It should prioritize and increase PPEs and other sundries to all staff categories at the frontline:

But if those guys can increase the supplies to us, then I think we can do better. We are doing well but I think we can do better. Supplies especially PPEs should be given enough priority. May be we get a surplus budget or something else. (FGD_Covid Frontline Workers_COVID-19 Treatment Unit Mulago Hospital_P1).

I look at the resources, I think it's high time the government should consider WHO's guidelines on allocation of funds for infrastructure. Currently, our budget has been standing at $7.5 \%$ for the health sector. Let's increase to at least 15\% so that facilities are able to handle any emerging or re-emerging cases at any time without running to donors. (FGD_ Covid Frontline Worker Entebbe Regional Referral Hospital_P3).

Rectify salary and risk allowance delays, avail health insurance to frontline workers and compensate their family members to boast staff morale:

The government and ministry should re-strategize on how well to take care of the healthcare workers because if it was not us, a lot of dead bodies and graves would have been got and seen. (FGD_Covid Frontline Workers_COVID-19 Treatment Unit Mulago Hospital_P1).

Ministry should ensure allowances are given on time. If the money is not there, then it should not promise allowances to people.

Responsible government officials have to be reminded of the allowances for all the healthcare workers. They need to know that surely HCWs are risking their lives, although they are here as their profession demands, but they have families to care for. (FGD_Covid Frontline Workers_COVID-19 Treatment Unit Mulago Hospital_P1).

Psychosocial support including financial motivation, stress management and appreciation for frontline workers on ground was suggested:

At least they should not stress or disvalue the people on ground. Let them first mind their lives so that these people can be treated or handled well. (FGD_Covid Frontline Workers_COVID-19 Treatment Unit Mulago Hospital_P1). 


\subsection{Facility.}

Offering extensive trainings to all frontline workers, availing Continuing Professional Development (CPD) and constant reminders on donning (proper wearing of PPEs as well as how to behave around CTU):

Streamline the procurement process of the drugs and include the unavailable drugs into the essential medicine list. Each department can be availed with Petty cash to buy out of stock drugs:

Avail petty cash to use, what could be the alternative to that because we need to be able to find solutions for each problem individually from each department. (FGD_Covid Frontline Workers_COVID-19 Treatment Unit Mulago Hospital_P1).

Reduction in turnaround time for COVID-19 test results, innovate rapid diagnostic tests with shorter turnaround time:

Then also maybe the issues with laboratories (labs), the labs should find a way of reducing turnaround time for testing. I'm aware of some tests that don't take 24 hours before release of results, those are the ones that should be used. The lab should also increase on the follow up of the tests they take off from patients such that delay of receiving results by patients is somehow minimized. (FGD_Covid Frontline Workers_COVID-19 Treatment Unit Mulago Hospital_P1).

Are the lab technicians receiving the reagents they require to have short turnaround of results? Are they having everything they require so that they can be able to perform tests adequately? So if no let's supply that. (FGD_Covid Frontline Workers_COVID-19 Treatment Unit Mulago Hospital_P1).

Decongesting of hospitals was suggested. This could be done by opening up other treatment centers similar to Namboole stadium or encouraging patients to self-isolate at home:

We need to depopulate this center because a lot of asymptomatic people are here eating food, taking resources that could have been used for people who are critically ill. So, I suggest that asymptomatic patients should self-isolate while in their homes and not in hospitals. (FGD_COVID Frontline Workers_COVID-19 Treatment Unit Mulago Hospital_P3).

Increasing staff personnel at the laboratory and offering all staff health insurance was suggested by participants:

I think increasing the number of personnel would help because then I think everyone would be more productive, have shorter periods of work but very productive. Then, I've heard of/seen the UK model where you work for 2 weeks, and stay home for 2 weeks. I'm not sure how feasible that is but I'm sure it would make people productive all the time. (FGD_ Covid Frontline Worker Entebbe Regional Referral Hospital_P2).

\subsection{Community}


Ensure continuous community sensitization about PPE, hand washing, putting on masks correctly and consistently, maintaining social distance and home isolating in case of flu-like symptoms:

But then, the Ministry Of Health should take a step of faith and allow people to home isolate. Because hospitals are over stretched and by the look of things, the ministry doesn't have enough funds to give to hospitals. (FGD_Covid Frontline Workers_COVID-19 Treatment Unit Mulago Hospital_P1).

\section{Discussion}

On 13th July 2020, Amnesty International released a report that indicated over 3000 health workers had died after being infected with COVID-19 in 79 countries.(16) The same report documented cases on how health workers who were raising safety concerns regarding the COVID-19 response were facing retaliation including being arrested and threatened by their particular governments.(16) In Egypt for example, 9 healthcare workers were arrested between March and June, on accounts of spreading false news and terrorism.(16) This study used this opportunity to therefore assess the psychosocial well-being and job satisfaction of the Ugandan COVID-19 Frontline healthcare workers. The authors feel this is an important contribution to the literature as we have been unable to find any published work related to the Ugandan COVID-19 Frontline healthcare workers' experiences and how satisfied they are regarding their job description in these unprecedented times. Taking care of the Frontline HCWs psychosocial well-being is as important as taking care of their physical health.

The WHO Public health law elaborates that during any public health emergency, the main responsibility of Healthcare workers is the public's interests and health rights.(17) From our study, the respondents reported that despite the stress they are facing during the COVID-19 Pandemic, they have to fulfill the Hippocratic Oath which they swore. The Hippocratic Oath states that doctors have to always consider patient care first (18) however the World Medical Association stressed that doctors should prioritize their own health as well as that of their patients.(19) Since COVID-19 is unprecedented to many healthcare workers, every event should be counted as pertinent.

Ensuring HCWs are motivated in such a crisis is important. However, there isn't yet any universal theory that can clearly envisage what can keep people motivated. Findings from the present study showed that appreciating the healthcare workers greatly motivated them and felt that their work was valued. This finding is similar to one of the initiatives aimed at boosting morale of Health care workers in Singapore General Hospital whereby well-wishers used to send cards, gifts, and messages appreciating the work of the frontline HCWs.(20) However, a study done by Haozheng and colleagues, in Hunan province China revealed that the main factor in encouraging HCWs was re-assuring them of personal safety when working.(21)

Job satisfaction is an important motivational resource in preventing burnout during epidemics.(22) A study done by Zhang and colleagues in Iran revealed that access to Personal Protective Equipment (PPEs) was a predictor to job satisfaction and reduced distress.(23) An article written by Melanie Newman in Britain called the lack of adequate PPEs as "totally unacceptable" since it could lead to HCWs 
quitting and some dying.(24) From our study, all the respondents reported having inadequate PPEs and drug stock outs in their treatment centers thus putting them at a risk of being infected by COVID-19 from patients. This finding is similar to the study done in US and Pakistan which showed that HCWs in both countries had been coerced to work with no Personal Protective Equipment.(25) Because COVID-19 is spread primarily through droplets or contact and yet there is still no definite cure, the only frontline protection for HCWs is the PPE.(25)

From our study, majority of the respondents reported poor remuneration in terms of delayed or low salary compared to the risk they undertake thus causing dissatisfaction among most of them. This finding is not different from that of Hagopian and colleagues on Job satisfaction among Ugandan healthcare workers. The study by Hagopian revealed that only $11 \%$ of the participants thought their salary package was fair.(26) From our study, most of the participants had a feeling that their allowances and salaries were shadowed in some politician offices thus pointing to corruption as the major cause of the delayed and low salaries. The study by Hagopian had similar findings as well, whereby the participants thought corruption was among the main causes of the problem.(26) Inorder to solve this challenge, most of the respondents in this study suggested that the Ministry of Health and government of Uganda should avail health insurance to every frontline HCW and their families:

Health workers are some of the workers who don't have insurance yet they're in a high risk zone. It is unfortunate that we've lost some HCWs on the frontline but their families have not been compensated, which is also a demotivating factor to the other frontliners. Why should I risk my life when after tomorrow I'm away, my family will suffer for the rest of their life? It's also a factor to be looked into. (FGD_ Covid Frontline Worker Entebbe Regional Referral Hospital_P2).

From this current study, most of the participants reported having fear and anxiety of contracting COVID19 from patients and later on spreading it to the family members. In addition, due to isolation and avoidance of family and friends in a bid of preventing transmission, most of the participants reported being stressed out due to this action. This finding is not different from multiple studies carried out worldwide regarding the anxiety among frontline health workers. $(21,27,28)$ A study done by Jagdesh and colleagues in Pakistan revealed that the three most common factors contributing to the fear were: getting infected, being quarantined, and losing life (29) and as a result, majority of the participants (65\%) in this study were hesitant to work. From the published work so far, the fear of getting infected can be minimized by setting up Standard Operating Procedures (SOPs) like social distancing while talking to patients, donning of PPE appropriately, and hand hygiene. In addition there should be provision of adequate PPE and assurance of the HCWs on which kind of care and benefits they are to get after them and their families getting infected.

From our study, majority of the respondents suffered stigma and discrimination from community members. In addition, some of the respondents in our study reported discrimination of their family members especially children by the community members. An article written by Poudel from Nepal, revealed that several COVID-19 frontline health care workers were facing difficulties getting shelter and 
food due to social discrimination.(30) Similar occurrences have been seen in India where HCWs have been threatened, beaten and chased away from their homes.(31) In Mexico, HCWs were restricted from using public transport and thus had to use bicycles as the means of transport to health facilities.(31) This attitude exhibited by the community has created an environment not conducive in controlling this pandemic crisis and adding more burden to the HCWs. A professor of Microbiology in India attributed the stigma and discrimination to the infodemic of the virus in addition to the unscientific beliefs held by most of the people.(32) It is therefore crucial to disseminate accurate and detailed COVID-19 related information to the public, and active engagement of the public inorder to counteract the unscientific beliefs. An article written by Tandon indicated that indeed provision of accurate COVID-19 related information enables people to make appropriate decisions regarding their roles in fighting this pandemic. (33) World Health Organisation (WHO) encourages a 'people first' language while communicating as a strategy of combating stigma.(34)

\section{Strengths and Limitations.}

The use of FGDs facilitated the understanding of the COVID-19 Frontline HCWs experiences and suggestions to some of the challenges they were facing. The inclusion of all categories of profession involved in the management of COVID-19 helped in capturing experiences every profession had. Indeed, doctors, nurses, hygienists, laboratory personnels, and security operatives could not all have the same experiences, therefore with this study experiences and challenges to every cadre was captured. However, our findings should be taken with caution due to the following limitation: Socially acceptable responses could have been given by the respondents due to lack of privacy. The FGDs were done in a single room containing all the 5 participants per FGD. Therefore due to fear of being reported by their fellow coworkers or bosses, respondents could have given socially acceptable responses. However, there was an attempt of minimizing this by ensuring the respondents that their responses will be kept confidential and each respondent was made to sign a confidential agreement restricting them from disseminating what is discussed in the FGD to other third parties.

\section{Conclusion}

In conclusion, working in a setting with patients having a disease of high infectivity creates remarkable stigmatization. The study found out that healthcare workers were remarkably stressed, exhausted and burnt-out due to the heavy workload and inadequate personal protective Equipment. In addition, they faced social stigma and discrimination from the community coupled with the dissatisfaction due to the low and delayed salary from the government. Hospitals should therefore create conducive environments that support the Healthcare workers' efforts positively. Counselling services for HCWs are recommended to be introduced into hospitals inorder to combat the issue of social stigma and its harmful effects. There should be adequate remuneration of the HCWs according to the risk they are undertaking and provision of healthcare insurance to them and their family as a protective mechanism in case they get infected is recommended. 


\section{Abbreviations}

COVID-19: Corona Virus Disease 2019, HCWs: Healthcare Workers, MOH: Ministry of Health, FGDs: Focus group discussions, PPE: Personal Protective Equipment, CTU: COVID-19 Treatment Unit, ERRH: Entebbe Regional Referral Hospital, MNRH: Mulago National Referral Hospital, UK: United Kingdom, CPD: Continuing Professional Development, P1: Participant 1..., ICU: Intensive Care Unit, WHO: World Health Organisation

\section{Declarations}

\section{ACKNOWLEDGEMENT}

The authors would like to acknowledge Infectious Disease Institute Makerere University for the support rendered to this study.

The authors would also like to acknowledge Mulago National Referral hospital Research Committee for granting us with the approval for the study.

We extend our sincere gratitude to the administrators and the participants of the two facilities ERRH and MNRH where the study took place.

\section{FUNDING}

The study was funded by the CAPA-CT II Project which is part of the EDCTP2 programme supported by the European Union.

\section{AUTHORS:}

NT: Nelson Twinamasiko, AG: Anna Maria Gwokyalya, IN: Innocent Nakityo, AT: Adelline Twimukye, EW: Enoch Wasswa, ES: Emmanuel Sserunjogi, PR: Pius Rwamafa, ML: Mohammed Lamorde, HM: Harriet Kizza Mayanja.

\section{AUTHORS CONTRIBUTION.}

All authors made a significant contribution to the work reported, whether that is in the conception, study design, execution, acquisition of data, analysis and interpretation. They all took part in drafting, revising or critically reviewing the article; gave final approval of the version to be published; have agreed on the journal to which the article has been submitted; and agree to be accountable for all aspects of the work.

\section{CONFLICT OF INTEREST.}

The authors declare that the research was conducted in the absence of any commercial or financial relationships that could be construed as a potential conflict of interest. 


\section{AVAILABILITY OF DATA AND MATERIALS}

The FGD guide is attached in additional file 1. Source data is in recorded voice which could break anonymity of the participants. The FGDs were anonymized and transcribed. Because of the sensitive nature of this material, it will not be made available online, but the corresponding author can be contacted to discuss sharing of this data on a case by case basis.

\section{References}

1. Wu F, Zhao S, Yu B, Chen Y-M, Wang W, Song Z-G, et al. A new coronavirus associated with human respiratory disease in China. Nature. 2020;579(7798):265-9.

2. Zhou P, Yang X-L, Wang X-G, Hu B, Zhang L, Zhang W, et al. A pneumonia outbreak associated with a new coronavirus of probable bat origin. Nature. 2020;579(7798):270-3.

3. Riou J, Althaus CL. Pattern of early human-to-human transmission of Wuhan 2019 novel coronavirus (2019-nCoV), December 2019 to January 2020. 2020;25(4):2000058.

4. Tang D, Tou J, Wang J, Chen Q, Wang W, Huang J, et al. Prevention and control strategies for emergency, limited-term, and elective operations in pediatric surgery during the epidemic period of COVID-19. 2020;3(1).

5. Badahdah AM, Khamis F, Al Mahyijari NJPR. The psychological well-being of physicians during COVID-19 outbreak in Oman. 2020;289:113053.

6. Ives J, Greenfield S, Parry JM, Draper H, Gratus C, Petts JI, et al. Healthcare workers' attitudes to working during pandemic influenza: a qualitative study. 2009;9(1):1-13.

7. Wang C, Pan R, Wan X, Tan Y, Xu L, Ho CS, et al. Immediate psychological responses and associated factors during the initial stage of the 2019 coronavirus disease (COVID-19) epidemic among the general population in China. 2020;17(5):1729.

8. Maunder RG, Leszcz M, Savage D, Adam MA, Peladeau N, Romano D, et al. Applying the lessons of SARS to pandemic influenza. 2008;99(6):486-8.

9. Khalid I, Khalid TJ, Qabajah MR, Barnard AG, Qushmaq IAJCm, research. Healthcare workers emotions, perceived stressors and coping strategies during a MERS-CoV outbreak. 2016;14(1):7-14.

10. da Silva JV, Carvalho IJTPJ. Physicians experiencing intense emotions while seeing their patients: what happens? 2016;20(3).

11. Uganda MoH. COVID-19 Updates 2020 [Available from: https://www.health.go.ug/covid/documentcategory/updates/.

12. Lapolla P, Mingoli A, Lee RJIC, Epidemiology H. Deaths from COVID-19 in healthcare workers in ItalyWhat can we learn? 2020:1-2.

13. Xiong Y, Peng LJTLGH. Focusing on health-care providers' experiences in the COVID-19 crisis. 2020;8(6):e740-e1. 
14. Lai J, Ma S, Wang Y, Cai Z, Hu J, Wei N, et al. Factors associated with mental health outcomes among health care workers exposed to coronavirus disease 2019. 2020;3(3):e203976-e.

15. Kitzinger J, Barbour R. Developing focus group research: politics, theory and practice: Sage; 1999.

16. International A. Global health workers silenced, exposed and attacked 2020 [Cited on December 1 2020]. Available from: Available:https://www.amnesty.org/en/latest/news/2020/07/health-workersrights-covid-report/

17. Emergencies WPH. Health systems and Health law 2020 [cited 2020 December 1]. Available from: https://www.who.int/healthsystems/topics/health-law/chapter11.pdf

18. Miles SH. The Hippocratic Oath and the ethics of medicine: Oxford University Press; 2005.

19. Zealand RN. NZ Doctor's Hippocratic Oath Change Passed 2020 [cited 2020 December 1]. Available from: https://www.rnz.co.nz/news/world/341592/nz-doctor-s-hippocratic-oath-change-passed.

20. Lateef FJJoE, Trauma, Shock. Face to face with coronavirus disease 19: Maintaining motivation, psychological safety, and wellness. 2020;13(2):116.

21. Cai H, Tu B, Ma J, Chen L, Fu L, Jiang Y, et al. Psychological Impact and Coping Strategies of Frontline Medical Staff in Hunan Between January and March 2020 During the Outbreak of Coronavirus Disease 2019 (COVID-19) in Hubei, China. 2020;26:e924171-1.

22. Zhang SX, Huang H, Wei FJPR. Geographical distance to the epicenter of Covid-19 predicts the burnout of the working population: Ripple effect or typhoon eye effect? 2020:112998.

23. Zhang SX, Liu J, Jahanshahi AA, Nawaser K, Yousefi A, Li J, et al. At the height of the storm: Healthcare staff's health conditions and job satisfaction and their associated predictors during the epidemic peak of COVID-19. 2020.

24. Newman MJBBMJ. Covid-19: doctors' leaders warn that staff could quit and may die over lack of protective equipment. 2020;368.

25. Ahmed J, Malik F, Arif TB, Majid Z, Chaudhary MA, Ahmad J, et al. Availability of personal protective equipment (PPE) among US and Pakistani doctors in COVID-19 pandemic. 2020;12(6).

26. Hagopian A, Zuyderduin A, Kyobutungi N, Yumkella FJHa. Job Satisfaction And Morale In The Ugandan Health Workforce: The Ministry of Health must focus on ways to keep health care workers from leaving their jobs-or leaving the country altogether. 2009;28(Suppl1):w863-w75.

27. Taghizadeh F, Hassannia L, Moosazadeh M, Zarghami M, Taghizadeh H, Dooki AF, et al. Anxiety and Depression in Health Workers and General Population During COVID-19 Epidemic in IRAN: A WebBased Cross-Sectional Study. 2020.

28. Mohindra R, Suri V, Bhalla A, Singh SMJAJP. Issues relevant to mental health promotion in frontline health care providers managing quarantined/isolated COVID19 patients. 2020;51:102084.

29. Kumar J, Katto MS, Siddiqui AA, Sahito B, Ahmed B, Jamil M, et al. Predictive Factors Associated With Fear Faced by Healthcare Workers During COVID-19 Pandemic: A Questionnaire-Based Study. 2020;12(8). 
30. Poudel AJAhkcns-a-h-w-a-a-l-c-i-c-hAJ. Stigma Against Health Workers, Patients and Area Locals Continues in Covid-19 Hotspots. 2020;15:2020.

31. Withnall AJTI. Coronavirus: why India has had to pass new law against attacks on healthcare workers. 2020.

32. Bagcchi SJTLID. Stigma during the COVID-19 pandemic. 2020;20(7):782.

33. Tandon RJAjop. The COVID-19 pandemic, personal reflections on editorial responsibility. 2020;50:102100.

34. Organisation WH. Social stigma associated with COVID-19 2020 [cited 2020 December 3]. Available from: https://www.who.int/docs/default-source/coronaviruse/covid19-stigma-guide.pdf.

\section{Supplementary Files}

This is a list of supplementary files associated with this preprint. Click to download.

- Additionalfile1.docx 